\title{
Colorimetric Detection of Perfluorinated Compounds by All-Polymer Photonic Transducers
}

\author{
Paolo Giusto, ${ }^{\dagger, \|}$ Paola Lova, ${ }^{\dagger}$ Giovanni Manfredi, ${ }^{\dagger}$ Serena Gazzo, ${ }^{\dagger}$ Padmanabhan Srinivasan, ${ }^{\ddagger}$, \\ Stefano Radice, ${ }^{+}$and Davide Comoretto ${ }^{*}, \oplus$ \\ ${ }^{\dagger}$ Dipartimento di Chimica e Chimica Industriale, Università di Genova, via Dodecaneso 31, 16126 Genova, Italy \\ ${ }^{\ddagger}$ Solvay Specialty Polymers, Viale Lombardia 20, Bollate, 20121 Milan, Italy
}

\section{Supporting Information}

ABSTRACT: We report on the highly sensitive optical and colorimetric detection of perfluorinated compounds in the vapor phase achieved by all-polymer dielectric mirrors. High optical quality and uniformly distributed Bragg reflectors were fabricated by alternating thin films of poly( $N$-vinylcarbazole) and Hyflon $\mathrm{AD}$ polymers as high and low refractive index medium, respectively. A new processing procedure has been developed to compatibilize the deposition of poly $(N$-vinylcarbazole) with the highly solvophobic Hyflon $\mathrm{AD}$ polymer layers to achieve mutual processability between the two polymers and fabricate the devices. As a proof of principle, sensing measurements were performed using the Galden HT55 polymer as a prototype of the perfluorinated
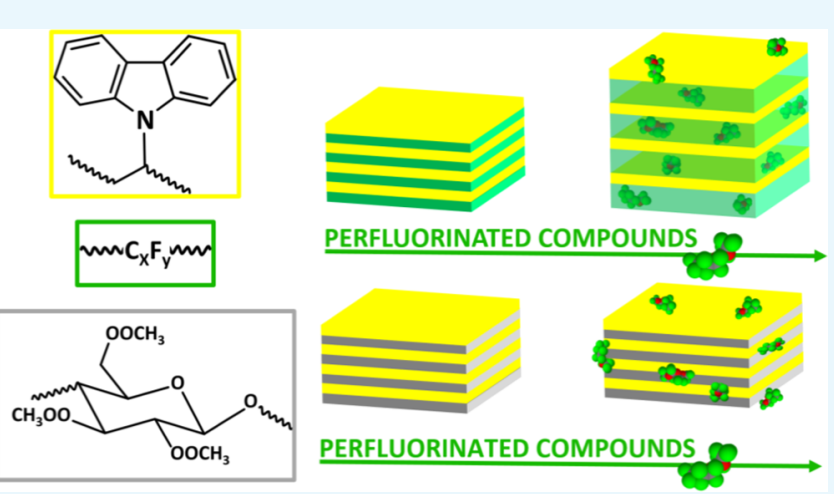
compound. The Bragg stacks show a strong chromatic response upon exposure to this compound, clearly detectable as both spectral and intensity variations. Conversely, Bragg mirrors fabricated without fluorinated polymers do not show any detectable response, demonstrating that the Hyflon $\mathrm{AD}$ polymer acts as the active and selective medium for sensing perfluorinated species. These results demonstrate that organic dielectric mirrors containing perfluorinated polymers can represent an innovative colorimetric monitoring system for fluorinated compounds, suitable to improve both environmental safety and quality of life.

\section{INTRODUCTION}

Nowadays, fluorinated products and polymers offer a wide range of materials with outstanding performances and technological solutions for high demanding applications. These applications very often constitute challenges in many key sectors like automotive, aeronautics, healthcare, energy, storage, and many others. Properties related to performances may include chemical inertness, thermal resistance, abrasion and weathering protection, water and stain repellency, biocompatibility, transparency, and many others. ${ }^{1,2}$ Usually, these materials are soluble only in expensive fluorinated solvents, whereas their nonwettability prevents the adhesion of overcoatings and makes them difficult to manage together with nonfluorinated compounds. Moreover, the detection of perfluorinated molecules (PFCs) is an important task, which makes any progress to increase the capability to assess the presence of these molecules highly interesting to monitor and preserve environmental health and safety. In this respect, a simple method for the assessment of air containing volatile PFCs using cost-effective sensors is of great importance. Optical and chromatic measurements constitute a class among simple methods and has been demonstrated in a variety of structures including molecular systems, ${ }^{3}$ photonic crystals, ${ }^{4,5}$ and liquid crystalline nanostructures. ${ }^{6-8}$ In particular, polymer dielectric mirrors (or distributed Bragg reflectors, DBR) proved to be a reliable and low-cost platform for colorimetric sensing for both organic and inorganic analytes in the vapor phase. $^{9-13}$ These devices are made of polymer layers having a different refractive index periodically alternated to generate dielectric submicrometric lattices. The interaction between light and these dielectric lattices, which are also known as monodimensional or planar photonic crystals, induces frequency regions forbidden to photon propagation. These frequencies are called photonic band gaps (PBGs) and are detectable as bright colors with the naked eyes, and as maxima of reflectance in the DBR spectrum. Polymer-DBR sensors are based on the intercalation of an analyte within the polymer layers. This process swells the polymers increasing their thickness, and thus, affecting the dielectric lattice. Such swelling is reflected in the DBR spectra as a shift of the PBG. The overall process can be driven by several analytepolymer interaction mechanisms. For instance, the kinetics of intercalation of an analyte within the polymer DBR can be engineered by modifying the free volume between the polymer chains using nanocomposite structures. ${ }^{2}$ Another mechanism exploits the formation of the guest-induced crystalline clathrate

Received: March 23, 2018

Accepted: June 19, 2018

Published: July 9, 2018 
domains in amorphous polymers. This mechanism, combined with different chemical affinity for different polymer-analyte pairs, plays a role in label-free molecular recognition. ${ }^{4}$ Because the interaction between the analyte and the polymers was demonstrated to be critical to govern the sensitivity and the selectivity of the DBR sensors, here, we propose the use of a perfluorinated polymer (PFC-P, copolymer of tetrafluoroethylene and 2,2,4-trifluoro-5-trifluoromethoxy 1,3-dioxole, known commercially as the Hyflon $\mathrm{AD}$ polymer, by Solvay Specialty Polymers) for the detection of PFCs. In addition to its affinity for PFCs, this polymer possesses a very low refractive index $(\sim 1.33)$, which allows a remarkable increase in the dielectric contrast with respect to polymer pairs commonly used to grow DBRs. Indeed, this parameter usually suffers from drawbacks related to the polymer mutual processability connected to the requirement for high transparency. ${ }^{14}$ The large dielectric contrast implies that high reflectance values can be obtained using a relatively small number of bilayers, thus, saving materials and making the device fabrication faster. ${ }^{14}$ However, as any other PFC, these polymers are highly solvophobic and scarcely wettable. This implies low mutual processability with other polymers. As a consequence, despite their optical transparency and low refractive index, very few examples of DBRs made of PFC-P have been reported in the literature so far. ${ }^{15-18}$

In this work, we take advantage of our expertize in processing PFC- $\mathrm{P}^{19,20}$ to design and fabricate relatively homogeneous and large area Hyflon $\mathrm{AD}$ polymers $(\mathrm{HY})$ :poly(N-vinylcarbazole) (PVK) DBRs as a proof of concept sensing devices. Galden HT55, perfluoropolyether polymer, was used as a prototype analyte of PFCs. Our results offer a simple colorimetric detection of PFCs in the vapor phase for the first time, suggesting a new and simple approach to easy environmental detection of PFCs.

\section{RESULTS AND DISCUSSION}

Due to low surface energy and solvophobicity, it is difficult to implement HY films into multilayered structures. Indeed, for the growth of DBRs, the deposition of polymer thin films need a tight control of thickness and interface roughness, which should not exceed few nanometers over large areas. ${ }^{21}$ To make HY layers suitable for the deposition of PVK films, their surface was activated by room temperature plasma treatment. Such treatment results in improved adhesion properties. ${ }^{22-24}$ To develop a routine for $\mathrm{HY}$ activation, we tested plasmas with different $\mathrm{O}_{2} / \mathrm{N}_{2}$ ratios. ${ }^{19}$ The surface activation was assessed by water contact angle measurements for over $24 \mathrm{~h}$ after the plasma treatment. Figure 1 reports the contact angles measured before and after the treatments. Before the treatment (black dot in Figure 1), the sample shows a contact angle of $119 \pm 1^{\circ}$. Supporting Information Figure S1 reports the value collected for nine sample cast using different conditions, demonstrating the reliability of the growth procedure. Upon surface treatment, the contact angle dramatically decreases to an upper value of $\sim 103^{\circ}$ for $\mathrm{O}_{2}$ plasma, and a lower value of $97^{\circ}$ for air-plasma (colored dots in Figure 1). $\mathrm{N}_{2}$ and different $\mathrm{O}_{2} / \mathrm{N}_{2}$ mixtures induce instead contact angles between these limit values. The surface activation was then monitored for 24 $\mathrm{h}$ by storing the samples in a petri dish in room conditions without any specific precaution. The data in Figure 1 show that only small variations occur when the samples are treated with all the gas mixtures but air, where the contact angle further decreases over the $24 \mathrm{~h}$. These results indicate that the surface

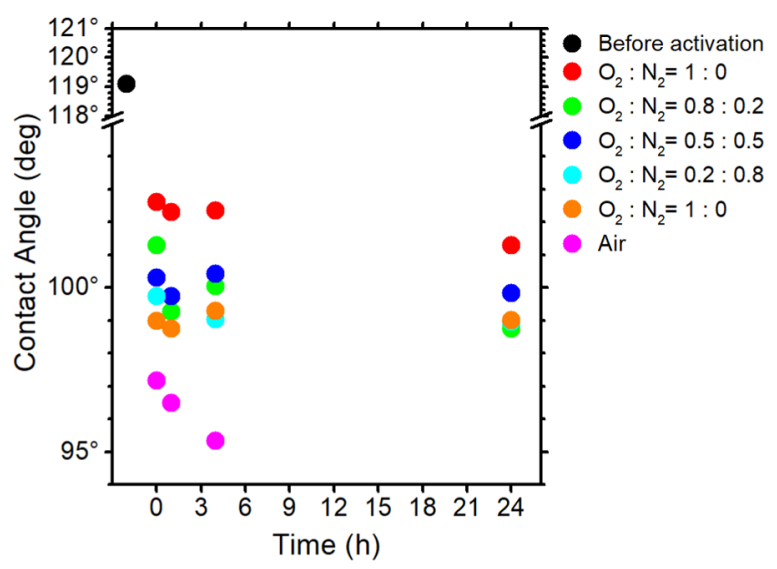

Figure 1. Average water contact angle of bare $\mathrm{HY}$ films as retrieved from Supporting Information Figure S1 (black dot), and after 0, 1, 4, and $24 \mathrm{~h}$ since the plasma treatment with different $\mathrm{O}_{2} / \mathrm{N}_{2}$ mixtures (colored dots).

of the HY film remains wettable over time, suitable for the preparation of multilayered structures. In this regard, $\mathrm{N}_{2}$ plasma induces the lowest contact angle stable on the longer time scale, and was then employed as routine treatment during the DBR fabrication. ${ }^{25}$

DBRs were then grown on 1 in. 2 glass substrate starting from the deposition of a PVK layer. After PVK deposition and thermal annealing, a layer of $\mathrm{HY}$ was cast on the latter and the surface activation was performed. This operation was repeated seven times to grow the multilayer. Figure $2 \mathrm{a}, \mathrm{b}$ show the
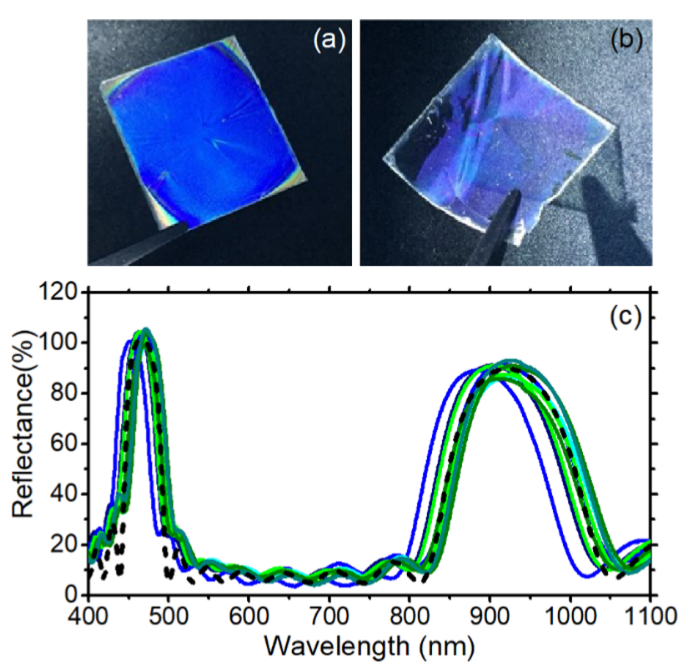

Figure 2. (a, b) Photographs and (c) experimental (continuous lines) and calculated (dashed line) reflectance spectra of HY:PVK DBR.

photographs and Figure $2 \mathrm{c}$ shows the spectra of a DBR sample resulting from this routine. Figure 2 a displays the sample as cast on the glass substrate, which appears blue and highly relatively homogeneous. The photograph in Figure $2 \mathrm{~b}$ reports instead a similar sample after peeling-off from the substrate. The Figures demonstrate that highly homogeneous, freestanding, and flexible DBRs can be fabricated with the method we propose. The DBR homogeneity was also confirmed by the reflectance spectra of the sample collected over six different spots of its surface. Indeed, Figure $2 c$ shows that the peak intensity and spectral position oscillates within 2 and $4 \%$, 

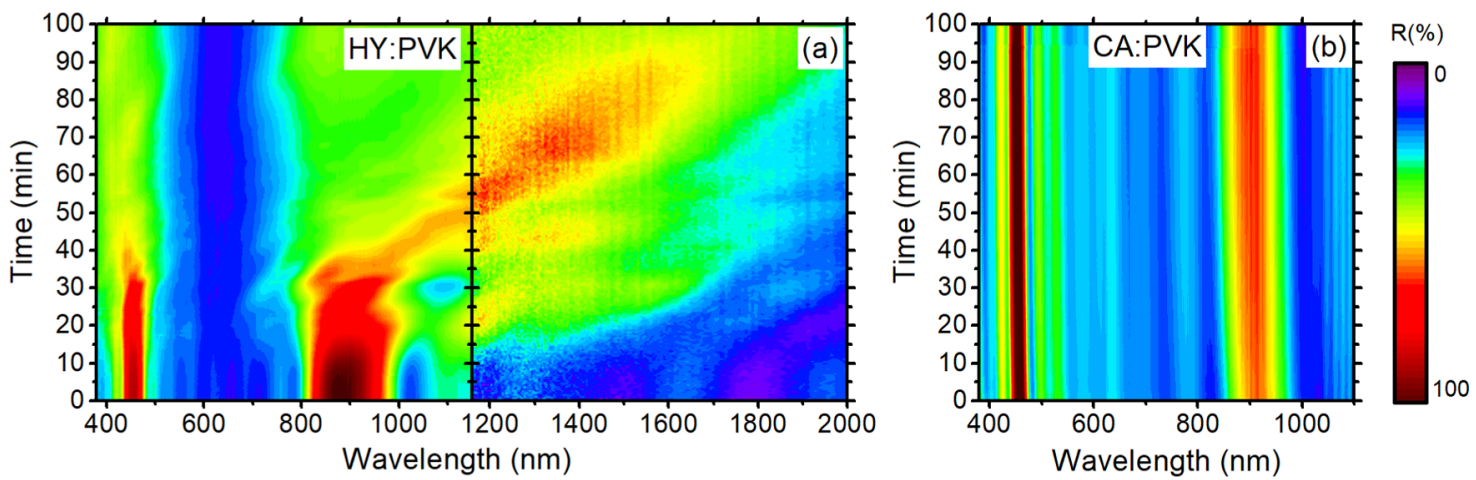

Figure 3. Contour plot of the (a) HY:PVK and (b) cellulose acetate (CA):PVK DBR spectra collected during the exposure to Galden HT55 polymer saturated vapors.

respectively. The spectra also show a well-defined interference pattern in the background, demonstrating very high quality of the top and bottom surfaces of the sample. ${ }^{2}$ The spectra show a prominent reflectance peak at $\sim 900 \mathrm{~nm}$ assigned to the PBG and a second order diffraction peak at $\sim 450 \mathrm{~nm}$, responsible for the brilliant blue color observed in the pictures reported in Figure 2a,b. The very high reflectance intensity of the PBG is due to the large dielectric contrast achieved with HY and PVK, which is the largest reported so far for polymer DBR grown by commercial polymers. The experimental spectra can be nicely reproduced with those calculated via a transfer matrix method (dashed black line in Figure 2c $)^{26,27}$ using layer thicknesses $d_{\mathrm{Hy}}$ $=102 \pm 4 \mathrm{~nm}$ and $d_{\mathrm{PVK}}=196 \pm 3 \mathrm{~nm}$ and refractive index values $d_{\mathrm{Hy}}=1.33^{28}$ for $\mathrm{HY}$ and $n_{\mathrm{PVK}}$ as retrieved in previously published data. ${ }^{26}$ The thickness uncertainty is comparable to the interlayer roughness investigated in a previous work by $\mathrm{X}$ ray reflectivity. ${ }^{21}$

The sensitivity of the HY:PVK DBRs to fluorinated compounds was assessed exposing the sample to the Galden HT55 polymer for 100 min. Figure 3a displays the contour plot of the reflectance spectra collected as a function of the exposure time. Here, the PBG is observed in red tones, whereas the spectra background is in blue shades. In the initial stages of exposure, the first and second order PBGs are detected at 900 and $450 \mathrm{~nm}$ respectively. These spectral features remain unchanged for the first $20 \mathrm{~min}$. According to models reported in a previous work, ${ }^{2}$ this induction time is assigned to the analyte permeation across the first PVK layer and can be increased or decreased, changing its thickness. To demonstrate this statement, Supporting Information Figure S2 shows that when the DBR is capped with HY layers instead of PVK, the induction time is reduced within the time resolution of our system (few milliseconds). After the induction time, the DBR spectrum undergoes a dramatic change. The first order PBG quickly fades while shifting toward longer wavelengths. Upon $50 \mathrm{~min}$ of exposure, it is shifted at about $1100 \mathrm{~nm}$. Upon longer exposures, the feature keeps shifting deeper in the near infrared part of the spectrum and inhomogeneously broadens. This behavior occurs until $90 \mathrm{~min}$ of exposure, when the PBG intensity decreases, and the feature cannot be distinguished clearly from the background of the spectrum anymore. The spectral details of the optical response of bare and long time exposed DBRs are reported in Supporting Information Figure S3. These characteristics have already been observed for different analyte/all-polymer DBR systems, ${ }^{2,4}$ and are assigned to the large intake of the analyte, which largely swells the active polymer, until the order and the periodicity of the sample get destroyed and the reflectance intensity of the $\mathrm{PBG}$ is dramatically reduced. Concerning the second order PBG, at $450 \mathrm{~nm}$, the glass substrate and the PVK absorptions reduce the effects of the analyte intercalation in the optical response. The diffraction peak remains indeed unchanged for about 20 min of exposure, and then its intensity fades indicating the loss of periodicity order of the structure. The behavior just observed can be interpreted considering a progressive intercalation of the analyte through the DBR surface closer to the enriched environment, ${ }^{2}$ and thus, a progressive and inhomogeneous swelling of the HY layers starting from the one on the top of the DBR, which is closer to the analyte environment, to the bottom one, which is closer to the glass substrate. Under this assumption, we simulated the optical response of the sample after $80 \mathrm{~min}$ of exposure considering the DBR layers to be closer to the unswollen substrate, and a random normal distribution of the thicknesses of the upper $\mathrm{HY}$ layers $(295 \pm 133 \mathrm{~nm}$, see Supporting Information Figure S3 for details). The good agreement between the experimental and calculated spectra (Supporting Information Figure S3) confirms the hypothesis that the analyte intercalation generates a giant swelling of the $\mathrm{HY}$ layers combined with a great amount of disorder. These data also agree with our previous findings on different all-polymer DBR exposed to selected analytes, and suggest that HY-based sensing devices may be suitable to reveal and discriminate different vapor analytes with a lower detection limit at the ppm level and sensitivity of $\mathrm{ppb}^{2,4}$

To prove the role of $\mathrm{HY}$ in the detection of the perfluorinated compounds, we compared the response to perfluorinated analytes of CA:PVK DBRs, which are known to be very efficient in the analysis of aromatic, heteroaromatic, or alcoholic species and water. ${ }^{2,4,6}$ Figure $3 \mathrm{~b}$ shows the response of CA:PVK DBR to Galden HT55 polymer vapors. No effects, even minor, are observed on the spectral position and the intensity of the PBGs over $100 \mathrm{~min}$ of exposure. This demonstrates that the fluorinated analyte does not interact with the CA and PVK.

The analysis of the combined optical response to Galden vapors clearly indicates that only the presence of a perfluorinated polymer like the Hyflon $\mathrm{AD}$ polymer in the DBR allows the detection of the PFC analytes as sketched in Figure 4. Here, the fluorinated Hy films undergoes strong swelling upon intercalation of Galden HT55 (Figure 4a), inducing a strong bathochromic shift of the PBG spectral position observed in Figure 3a. Conversely, when CA is used as a low index medium, the chemico-physical interaction between 


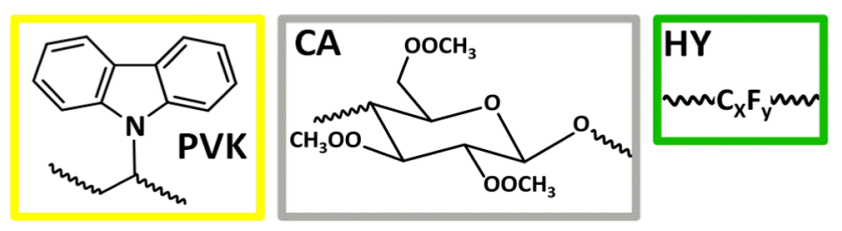

(a)

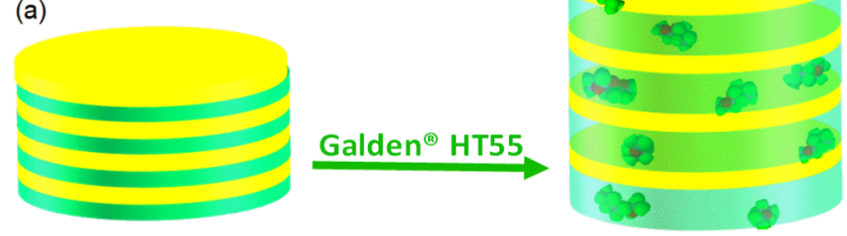

(b)

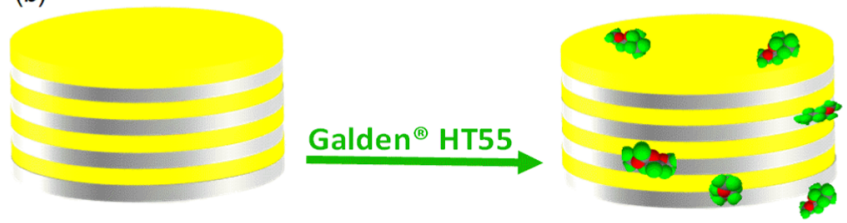

Figure 4. Schematic illustration of the sensing principle: (a) HY:PVK and (b) CA:PVK DBR exposed to Galden HT55. PVK is represented in yellow, CA in gray, and Hy in green. Galden HT55 representation is purely indicative.

the fluorinated analytes and the hydrogen-based polymer forbid the polymer swelling, and thus, the optical response (Figures $3 c$ and $4 b$ ). To the best of our knowledge, this is the first report on optical detection of fluorinated persistent volatile species by polymer photonic crystals.

Finally, we would like to comment on the design of the DBR sensor. Commonly, DBRs applied to photonics for lasing or for light emission control are designed to have layer thicknesses $d_{1} n_{1}=d_{2} n_{2}=\lambda_{\mathrm{PBG}} / 4,{ }^{29,30}$ where, $n$ is the refractive index of each layer and $\lambda_{\mathrm{PBG}}$ is the PBG spectral position. Such values satisfy the lambda fourth conditions, ${ }^{31}$ where the even orders of diffraction are not detectable in the DBR spectra, and odd PBGs with the highest reflectance and widest full width half maximum are achieved. However, high reflectance and bandwidth hinder the detection of small spectral variation. ${ }^{6}$ Besides this aspect, as previously reported by this group, ${ }^{2,4}$ the presence of a second order PBG tuned in the visible part of the spectrum and coupled with a first order PBG tuned in the near infrared region allows slower, and thus, more sensitive responses due to the first order of diffraction. Nevertheless, the colorimetric response due to the second order is maintained, allowing both simple ON/OFF colorimetric detection and quantitative assessment of fluorinated chemical species.

This work represents a proof of concept for easy chromatic detection of novel relevant analytes. However, a deeper investigation of the figures of merit of the sensors, including the lower detection limit, sensitivity, and selectivity is necessary to develop disposable sensors easy to operate even without the use of a spectrometer by untrained users. Indeed, analyte intercalation through the polymer films induces a dramatic color change due to the PBG bathochromic shift. Then a chemically switchable ON/OFF system can also be engineered. Moreover, polymer DBRs are currently the only photonic crystals industrially fabricable on the square meter scale by available technology already used for food packaging, like co-extrusion or continuous melting. These features, joined with the size of the present sensors, which is down to few square millimeters, make low-cost disposable sensors highly interesting.

\section{CONCLUSIONS}

We demonstrated the fabrication of flexible and high quality all-polymer DBR sensors with an area of 1 in. 2 made by HY, a perfluorinated copolymer with a very low refractive index, and the high refractive index PVK, using a simple and cost-effective technique as spin-coating. The high dielectric contrast achieved using these polymers allows very high reflectance with less than 10 bilayers, thus, achieving highly sensitive DBR sensors with a remarkable material saving. These DBRs show an outstanding optical response to fluorinated species in the vapor phase. For this purpose, the Galden HT55 polymer as a test molecule was used to demonstrate for the first time that the PFCs can be easily and selectively detected even by an untrained user and without complicated laboratory equipment by a label-free photonic crystal. These characteristics together with the possibility of mass production by established technologies, provide a new class of disposable and functional polymer photonic structures. Such DBR can be used to improve environmental safety and human quality of life.

\section{EXPERIMENTAL SECTION}

The DBR sensors were cast by spin-coating using PVK (SigmaAldrich, $\left.M_{\mathrm{w}}=135000\right)$ as a high refractive index material and $\mathrm{HY}$ as a low refractive index material. To demonstrate the sensing mechanism, some reference sensors were also fabricated replacing $\mathrm{HY}$ with cellulose acetate (CA, SigmaAldrich, $\left.M_{\mathrm{n}}=50000\right)$ as the low index medium as reported in previous works. ${ }^{27,32-35}$ PVK was dissolved in toluene $(35 \mathrm{~g} /$ L), ${ }^{26} \mathrm{CA}$ was dissolved in 4-hydroxy-4-methyl-2-pentanone $(36 \mathrm{~g} / \mathrm{L})$, and HY was dissolved in the Galden HT110 polymer $(30-42 \mathrm{~g} / \mathrm{L})$. Polymer solutions were then spun-cast at a rotation speed between 20 and 120 revolutions per second to obtain thicknesses in the range 100-200 nm. Due to the low surface energy of $\mathrm{HY}$, after the deposition of each $\mathrm{HY}$ layer, low power $\mathrm{O}_{2}-\mathrm{N}_{2}$ mixtures vacuum plasma treatment $(15 \mathrm{~W}$, 30 s) was performed to improve PVK adhesion by using a Gambetti Colibri system. ${ }^{19}$ After deposition of each PVK layer, thermal annealing at $80^{\circ} \mathrm{C}$ for $45 \mathrm{~s}$ was performed.

The sensitivity of the DBR sensors to PCFs was assessed by exposing them to a saturated environment of the Galden HT55 polymer $\left(P_{\text {vapor }}=0.27 \mathrm{kPa}\right)$ and monitoring their dynamic spectral response. Reflectance spectra were collected with homemade setups previously described ${ }^{4}$ using an aluminum mirror as a reference. For this reason, the reflectance intensity (and not absolute reflectivity) can be slightly higher than $100 \%$. Contact angles have been measured by a Theta Lite optical tensiometer by Biolin Scientific ${ }^{36}$ using the sessile drop technique. $^{37}$

\section{ASSOCIATED CONTENT}

\section{S Supporting Information}

The Supporting Information is available free of charge on the ACS Publications website at DOI: 10.1021/acsomega.8b00554.

Contact angle data; exposure of a HY:PVK DBR having HY as the upper layer to Galden HT50; experimental evolution of the reflectance spectrum upon exposition to 
Galden HT55 for HY:PVK DBR; experimental and calculated spectra CA:PVK DBR (PDF)

\section{AUTHOR INFORMATION}

\section{Corresponding Author}

*E-mail: davide.comoretto@unige.it.

\section{ORCID}

Paola Lova: 0000-0002-5634-6321

Giovanni Manfredi: 0000-0003-0682-0731

Davide Comoretto: 0000-0002-2168-2851

\section{Present Address}

"Max Planck Institute of Colloids and Interfaces, Department of Colloid Chemistry, Am Mühlenberg 1, 14476 PotsdamGolm, Germany (P.G.).

Notes

The authors declare no competing financial interest.

${ }^{\S}$ Retired.

\section{ACKNOWLEDGMENTS}

P.L. acknowledges the European Union's Horizon 2020 research and innovation program under the Marie Sklodowska-Curie grant agreement No. 643238.

\section{REFERENCES}

(1) Lindstrom, A. B.; Strynar, M. J.; Libelo, E. L. Polyfluorinated compounds: Past, present, and future. Environ. Sci. Technol. 2011, 45, 7954-7961.

(2) CDC (Centers for Disease Control and Prevention), Fourth National Report on Human Exposure to Environmental Chemicals; Atlanta, GA, 2009.

(3) Wang, L.; Li, Q. Photochromism into nanosystems: towards lighting up the future nanoworld. Chem. Soc. Rev. 2018, 47, 10441097.

(4) Kittle, J. D.; Fisher, B. P.; Esparza, A. J.; Morey, A. M.; Iacono, S. T. Sensing Chemical Warfare Agent Simulants via Photonic Crystals of the Morpho didius Butterfly. ACS Omega 2017, 2, 8301-8307.

(5) Chen, C.; Dong, Z.-Q.; Shen, J.-H.; Chen, H.-W.; Zhu, Y.-H.; Zhu, Z.-G. 2D Photonic Crystal Hydrogel Sensor for Tear Glucose Monitoring. ACS Omega 2018, 3, 3211-3217.

(6) Wang, L.; Dong, H.; Li, Y.; Xue, C.; Sun, L.-D.; Yan, C.-H.; Li, Q. Reversible Near-Infrared Light Directed Reflection in a SelfOrganized Helical Superstructure Loaded with Upconversion Nanoparticles. J. Am. Chem. Soc. 2014, 136, 4480-4483.

(7) Wang, L. Self-activating liquid crystal devices for smart laser protection. Liq. Cryst. 2016, 43, 2062-2078.

(8) Ling, W.; Quan, L. Stimuli-Directing Self-Organized 3D LiquidCrystalline Nanostructures: From Materials Design to Photonic Applications. Adv. Funct. Mater. 2016, 26, 10-28.

(9) Lova, P.; Manfredi, G.; Boarino, L.; Comite, A.; Laus, M.; Patrini, M.; Marabelli, F.; Soci, C.; Comoretto, D. Polymer distributed Bragg reflectors for vapor sensing. ACS Photonics 2015, 2, 537-543. (10) Lova, P.; Soci, C. Nanoimprint lithography: toward polymer photonic crystals. Organic and Hybrid Photonic Crystals, 1st ed.; Springer: Cham, Switzerland, 2015; Vol. 1, p 493.

(11) Lova, P.; Bastianini, C.; Giusto, P.; Patrini, M.; Rizzo, P.; Guerra, G.; Iodice, M.; Soci, C.; Comoretto, D. Label-free vapor selectivity in poly(p-phenylene oxide) photonic crystal sensors. ACS Appl. Mater. Interfaces 2016, 8, 31941-31950.

(12) Lova, P.; Manfredi, G.; Boarino, L.; Laus, M.; Urbinati, G.; Losco, T.; Marabelli, F.; Caratto, V.; Ferretti, M.; Castellano, M.; Soci, C.; Comoretto, D. Hybrid $\mathrm{ZnO}$ :polystyrene nanocomposite for allpolymer photonic crystals. Phys. Status Solidi C 2015, 12, 158-162.

(13) Manfredi, G.; Mayrhofer, C.; Kothleitner, G.; Schennach, R.; Comoretto, D. Cellulose ternary photonic crystal created by solution processing. Cellulose 2016, 23, 2853-2862.
(14) Gazzo, S.; Manfredi, G.; Pötzsch, R.; Wei, Q.; Alloisio, M.; Voit, B.; Comoretto, D. High refractive index hyperbranched polyvinylsulfides for planar one-dimensional all-polymer photonic crystals. J. Polym. Sci., Part B: Polym. Phys. 2016, 54, 73-80.

(15) Convertino, A.; Valentini, A.; Ligonzo, T.; Cingolani, R. Organic-inorganic dielectric multilayer systems as high reflectivity distributed Bragg reflectors. Appl. Phys. Lett. 1997, 71, 732-734.

(16) Convertino, A.; Capobianchi, A.; Valentini, A.; Cirillo, E. N. M. A new approach to organic solvent detection: High-reflectivity bragg reflectors based on a gold nanoparticle/teflon-like composite material. Adv. Mater. 2003, 15, 1103-1105.

(17) Song, H.; Singer, K.; Lott, J.; Wu, Y.; Zhou, J.; Andrews, J.; Baer, E.; Hiltner, A.; Weder, C. Continuous melt processing of allpolymer distributed feedback lasers. J. Mater. Chem. 2009, 19, 75207524.

(18) Karaman, M.; Kooi, S. E.; Gleason, K. K. Vapor deposition of hybrid organic-inorganic dielectric bragg mirrors having rapid and reversibly tunable optical reflectance. Chem. Mater. 2008, 20, 22622267.

(19) Radice, S. V.; Gavezoti, P.; Simeone, G.; Albano, M.; Congiu, S.; Canazza, G.; Comoretto, D. Photonic Crystals, International Patent Number: PCT/EP2015/055590, March 20, 2014.

(20) Radice, S. V.; Srinivasan, P.; Comoretto, D.; Gazzo, S. OneDimensional Planar Photonic Crystals Including Fluoropolymer Compositions and Corresponding Fabrication Methods, International Patent Number: WO 2016/087439 A1, December 1, 2016.

(21) Unger, K.; Resel, R.; Czibula, C.; Ganser, C.; Teichert, C.; Jakopic, G.; Canazza, G.; Gazzo, S.; Comoretto, D. In Distributed Bragg Reflectors: Morphology of Cellulose Acetate and Polystyrene Multila, 6th International Conference on Transparent Optical Networks (ICTON 2014), Graz, Austria, 2014; pp 1-4.

(22) Vesel, A.; Kovac, J.; Primc, G.; Junkar, I.; Mozetic, M. Effect of $\mathrm{H}_{2} \mathrm{~S}$ plasma treatment on the surface modification of a polyethylene terephthalate surface. Materials 2016, 9, 1-14.

(23) Junkar, I.; Vesel, A.; Cvelbar, U.; Mozetič, M.; Strnad, S. Influence of oxygen and nitrogen plasma treatment on polyethylene terephthalate (PET) polymers. Vacuum 2009, 84, 83-85.

(24) Vesel, A.; Junkar, I.; Cvelbar, U.; Kovac, J.; Mozetic, M. Surface modification of polyester by oxygen- and nitrogen-plasma treatment. Surf. Interface Anal. 2008, 40, 1444-1453.

(25) Pascu, M.; Nicolas, D.; Poncin-Epaillard, F.; Vasile, C. Surface modification of PVDF by plasma treatment for electroless metallization. J. Optoelectron. Adv. Mater. 2006, 8, 1062.

(26) Lova, P.; Grande, V.; Manfredi, G.; Patrini, M.; Herbst, S.; Würthner, F.; Comoretto, D. All-polymer photonic microcavities doped with perylene bisimide J-aggregates. Adv. Opt. Mater. 2017, 5 , No. 1700523.

(27) Manfredi, G.; Lova, P.; Di Stasio, F.; Krahne, R.; Comoretto, D. Directional fluorescence spectral narrowing in all-polymer microcavities doped with $\mathrm{CdSe} / \mathrm{CdS}$ dot-in-rod nanocrystals. ACS Photonics 2017, 4, 1761-1769.

(28) Groh, W.; Zimmermann, A. What is the lowest refractive index of an organic polymer? Macromolecules 1991, 24, 6660-6663.

(29) Comoretto, D. Organic and Hybrid Photonic Crystals, 1st ed.; Springer International Publishing, 2015; p 497.

(30) Bellingeri, M.; Chiasera, A.; Kriegel, I.; Scotognella, F. Optical properties of periodic, quasi-periodic, and disordered one-dimensional photonic structures. Opt. Mater. 2017, 72, 403-421.

(31) Hayes, G. R.; Cacialli, F.; Phillips, R. T. Ultrafast study of spontaneous emission from conjugated polymer microcavities. Phys. Rev. B 1997, 56, 4798-4801.

(32) Canazza, G.; Scotognella, F.; Lanzani, G.; Silvestri, S. D.; Zavelani-Rossi, M.; Comoretto, D. Lasing from all-polymer microcavities. Laser Phys. Lett. 2014, 11, No. 035804.

(33) Fornasari, L.; Floris, F.; Patrini, M.; Canazza, G.; Guizzetti, G.; Comoretto, D.; Marabelli, F. Fluorescence excitation enhancement by bloch surface wave in all-polymer 1D photonic structure. Appl. Phys. Lett. 2014, 105, No. 053303. 
(34) Fornasari, L.; Floris, F.; Patrini, M.; Comoretto, D.; Marabelli, F. Demonstration of fluorescence enhancement via Bloch surface waves in all-polymer multilayer structures. Phys. Chem. Chem. Phys. 2016, 18, 14086-14093.

(35) Lova, P.; Cortecchia, D.; Krishnamoorthy, H. N.; Giusto, P.; Bastianini, C.; Bruno, A.; Comoretto, D.; Soci, C. Engineering the Emission of Broadband 2D Perovskites by Polymer Distributed Bragg Reflectors. ACS Photonics 2018, 867.

(36) Rudakova, A. V.; Maevskaya, M. V.; Emeline, A. V.; Bahnemann, D. W. Light-controlled $\mathrm{ZrO} 2$ surface hydrophilicity. Sci. Rep. 2016, 6, No. 34285.

(37) Jokinen, V.; Suvanto, P.; Franssila, S. Oxygen and nitrogen plasma hydrophilization and hydrophobic recovery of polymers. Biomicrofluidics 2012, 6, No. 016501. 